\title{
Fresnel Light Drag in a Coherently Driven Moving Medium
}

\author{
M. Artoni, ${ }^{1}$ I. Carusotto, ${ }^{2}$ G. C. La Rocca, ${ }^{3,2}$ and F. Bassani ${ }^{2}$ \\ ${ }^{1}$ I.N.F.M., L.E.N.S., Largo E. Fermi 2, 50125 Florence, Italy \\ ${ }^{2}$ I.N.F.M., Scuola Normale Superiore, Piazza dei Cavalieri 7, 56126 Pisa, Italy \\ ${ }^{3}$ Dipartimento di Fisica, "E. R. Caianiello," Università di Salerno, 84081 Baronissi (Sa), Italy
}

(Received 18 September 2000)

\begin{abstract}
We theoretically study how the phase of a light plane wave propagating in a resonant medium under electromagnetically induced transparency (EIT) is affected by the uniform motion of the medium. For cuprous oxide $\left(\mathrm{Cu}_{2} \mathrm{O}\right)$, where EIT can be implemented through a typical pump-probe configuration, the resonant probe beam experiences a phase shift (Fresnel-Fizeau effect) that may vary over a wide range of values, positive or negative, and even vanishing, due to the combined effects of the strong frequency dispersion and anisotropy both induced by the pump. The use of such a coherently driven dragging medium may improve by at least 1 order of magnitude the sensitivity at low velocity in optical drag experiments.
\end{abstract}

DOI: $10.1103 /$ PhysRevLett.86.2549

The phase velocity of light depends on whether light propagates in a moving or in a stationary medium. This effect, which gives rise to the familiar Fresnel light drag $[1,2]$, has been observed for the first time in Fizeau's flowing water experiment [3] and had a profound influence on the change of our perception [4] of the nature of space and time at the turn of the century.

We here anticipate that light drags can be made to vary over a rather wide range of values when a slab of coherently driven cuprous oxide $\left(\mathrm{Cu}_{2} \mathrm{O}\right)$ is used as a dragging medium. This originates from the steep electromagnetically induced transparency (EIT) dispersion [5,6] occurring at the $2 P$ exciton resonance in a typical pump and probe $\Lambda$ configuration [7]. Furthermore, in a sample rendered anisotropic by a suitable choice of the pump and probe polarizations, one can also make the light drag vanish over a broad range of probe frequencies depending on the pump parameters. This means that in a typical interferometric experiment no fringe shift would be observed for light propagating through a moving medium with respect to light propagating through the same medium at rest. Such a somewhat surprising conclusion holds for all velocities of experimental interest and has been found so far to hold only in the very different context of matter waves where a null drag has been observed for thermal neutrons traversing a nonresonant moving medium contained inside stationary boundaries [8].

Since Fizeau's experiment various other observations of light drags have followed in which different dragging media and diverse interferometric measurement techniques have been employed, but there still remains a formidable challenge to perform high-precision measurements of light drags; these, in fact, have not yet reached the level of accuracy of other tests of special relativity [9]. In order to perform a high-precision measurement of the Fresnel-Fizeau effect one needs high sensitivity to velocity induced phase shifts, which in turn sets a lower bound for the usable sample speeds. At the same time, in order to preserve high contrast of the interference fringe pattern, mechanical vi-
PACS numbers: $42.50 . \mathrm{Gy}, 03.30 .+\mathrm{p}$

brations from the sample movement have to be minimized. Owing to the high dispersion at the $2 P$-exciton resonance, where absorption is also largely quenched by quantum interference, this goal can be achieved by using a slab of coherently driven cuprous oxide $\left(\mathrm{Cu}_{2} \mathrm{O}\right)$ as a dragging medium: interferometric sensitivity at low drag velocities can be increased by at least 1 order of magnitude.

The dragging medium, in the form of a parallel-sided slab, induces an optical phase shift which we here calculate from first principles including the effects of frequency and angular dispersion. We take a slab moving in one arm of the interferometer with constant velocity $v$ with respect to an observer in the laboratory frame $S$. The relevant geometry is illustrated in Fig. 1. Phase shifts depend crucially on boundary conditions and our configuration, unlike in the historic work of Fizeau [3] where the phase

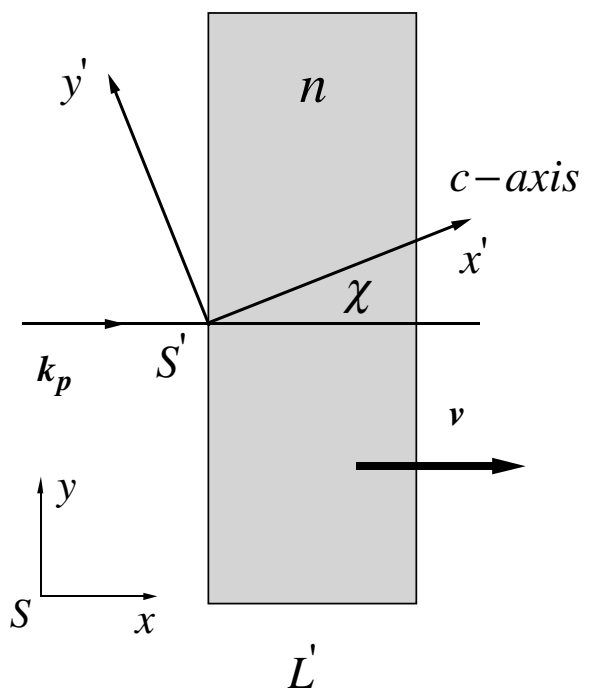

FIG. 1. Probe-beam path across a $\mathrm{Cu}_{2} \mathrm{O}$ slab moving with velocity $v$ in the laboratory frame $S$. The probe wave vector $\boldsymbol{k}_{p}$, the slab velocity, and its surface normal are all in the same direction $\hat{x}$. The pump wave vector is along $\hat{z}=\hat{z}^{\prime}$ and its polarization is parallel to the optical $c$ axis $\hat{x}^{\prime}$ which makes a fixed angle $\chi$ with $\hat{x}$. 
shifts were produced by water flowing inside stationary tubes, realizes a situation in which the medium boundaries are in motion [10]. Note that we do not account here for small tilt angles between the surface normal and the interferometer $\hat{x}$ axis commonly employed to minimize backreflections and to avoid multiple internal reflections [9]. Such a geometry is in fact sufficient to illustrate [11] the effects of frequency and angular dispersion associated with EIT on the light drag and we then proceed to derive the phase shift for a beam single-pass even if this occurs at normal incidence. The phase shift is in general associated with a space-time contour, i.e., $\Delta \phi=\oint(\omega d t-\boldsymbol{k} \cdot d \boldsymbol{r})$, which accounts for the temporal and spatial variations exhibited by a plane wave of wave vector $\boldsymbol{k}$ and angular frequency $\omega$ propagating in the medium. In our approach, we set all times to be identical in the laboratory frame $S$ and we evaluate $\oint \boldsymbol{k}_{p} \cdot d \boldsymbol{r}$ along the probe optical path in the interferometric arrangement required for a typical highprecision measurement of the drag $[9,12]$.

Because the phase shift is relativistically invariant, we find it convenient to derive its expression in the slab rest frame $S^{\prime}$. The space-time coordinates as well as the frequency $\left(\omega_{p}\right)$ and wave vector $\left(\boldsymbol{k}_{p}\right)$ of the probe light wave in $S$ and $S^{\prime}$ are connected through a Lorentz transformation [13] which comprises a boost along $x$ and a rotation about the $z$ axis. To the lowest order in $\beta=v / c$, the single pass phase shift is

$$
\begin{aligned}
\Delta \phi & =\Delta \phi^{\prime}=\omega_{p}^{\prime} \Delta t^{\prime}-\boldsymbol{k}_{p}^{\prime} \cdot \Delta \boldsymbol{r}^{\prime} \simeq-\beta \frac{\omega_{R}^{\prime} L^{\prime}}{c}-k_{R}^{\prime} L^{\prime} \\
& =-\frac{\omega_{R}^{\prime} L^{\prime}}{c}\left\{\operatorname{Re}\left[n\left(\omega_{R}^{\prime}, \chi\right)\right]+\beta\right\},
\end{aligned}
$$

where primed and unprimed variables refer, respectively, to the slab rest frame $S^{\prime}$ and the laboratory frame $S$ and where we have replaced $\omega_{p}^{\prime}$ by $\omega_{R}^{\prime}$ and $\boldsymbol{k}_{p}^{\prime}$ by $\boldsymbol{k}_{R}^{\prime}$ for the frequency and wave vector of the refracted probe beam propagating inside the slab. Here $n\left(\omega_{R}^{\prime}, \chi\right)$ denotes the complex index of refraction of the uniaxial medium in its rest frame $S^{\prime}$, while $L^{\prime}$ is the actual slab thickness.

Since the slab surfaces are stationary in $S^{\prime}$, there is no frequency change at the sample boundaries and the frequency of the refracted wave is equal to that of the incident one, i.e., $\omega_{R}^{\prime}=\omega_{I}^{\prime}$, while the incident probe frequency in $S$ and $S^{\prime}$ are related, to the lowest order in $\beta$, by

$$
\omega_{I}^{\prime} \simeq \omega_{I}-\beta \omega_{I} .
$$

Such a Doppler shift is quite small and the refractive index can then be expanded as

$$
n\left(\omega_{R}^{\prime}, \chi\right) \simeq n\left(\omega_{I}, \chi\right)-\beta \omega_{I} \frac{\partial n\left(\omega_{I}^{\prime}, \chi\right)}{\partial \omega_{I}^{\prime}},
$$

where the derivative is to be evaluated at $\omega_{I}^{\prime}=\omega_{I}$. Substituting (2) and (3) back into (1) yields

$$
\begin{aligned}
\Delta \phi= & -\frac{\omega_{I} L^{\prime}}{c} \operatorname{Re}\left[n\left(\omega_{I}, \chi\right)\right]-\beta \frac{\omega_{I} L^{\prime}}{c} \\
& \times\left[1-\operatorname{Re}\left[n\left(\omega_{I}, \chi\right)\right]-\omega_{I} \frac{\partial \operatorname{Re}\left[n\left(\omega_{I}, \chi\right)\right]}{\partial \omega_{I}}\right] \\
\equiv & \Delta \phi_{o}-\beta \frac{\omega_{I} L^{\prime}}{c} \alpha_{e} .
\end{aligned}
$$

This gives the relative phase of the incident and emerging beams as viewed in the laboratory frame to first order in $\beta$. Here $\Delta \phi_{o}$ is the shift induced by a stationary sample, while the additional term on the right hand side of (4) arises from the sample motion and defines in turn the effective drag coefficient $\alpha_{e}$ [9]. This is a measurable quantity as the nonreciprocal phase shift between the light beams propagating parallel and antiparallel to the velocity of the sample, that is proportional to $\alpha_{e}$, can easily be measured through standard high-precision interferometric techniques $[9,12]$. The usual definition [2] of the drag coefficient for the phase velocity of light can easily be related to the measurable quantity $\alpha_{e}$ [9].

We specifically evaluate the effective drag coefficient $\alpha_{e}$ experienced by a weak probe beam crossing a slab of $\mathrm{Cu}_{2} \mathrm{O}$ under EIT [7] used as a dragging medium; this is realized by tuning the probe about the $2 P$ yellow exciton line of resonant frequency $\omega_{2 P}$ and by further driving the sample with a strong pump beam of Rabi frequency $\Omega_{c}$ and frequency $\omega_{c}$ tuned to the $1 S-2 P$ exciton transition. The quadrupole allowed threefold degenerate $1 S$ exciton state $\left(\Gamma_{5}^{+}\right)$has a small linewidth $\left(\hbar \gamma_{1 S} \simeq 0.1 \mathrm{meV}\right)$ compared to that $\left(\hbar \gamma_{2 P} \simeq 1 \mathrm{meV}\right)$ of the second class dipole allowed threefold degenerate $2 P$-exciton state $\left(\Gamma_{4}^{-}\right)$. For a pump Rabi frequency $\Omega_{c} \approx \sqrt{\gamma_{2 P} \gamma_{1 S}}$ EIT takes place whereby a narrow transparency window associated with a rather steep dispersion appears about the $2 P$-exciton line [7]. The effective dielectric tensor describing the optical response of the medium to the weak probe in the presence of the pump can be derived from a $\Lambda$-type model Hamiltonian as developed in [7] and, in general, will be anisotropic. The specific form of the effective dielectric tensor depends on the detailed structure of the exciton levels involved and on the pump polarization. For the sake of simplicity, we here assume that the $\Gamma_{4}^{-}$states are well separated from all other $2 P$ states and we take the pump polarization along the cubic axis $\hat{x}^{\prime}$ (see Fig. 1). The resulting dielectric tensor is uniaxial with the optical $c$ axis along $\hat{x}^{\prime}$, i.e., $\epsilon_{x^{\prime} x^{\prime}}=\epsilon_{\|}$, $\epsilon_{y^{\prime} y^{\prime}}=\epsilon_{z^{\prime} z^{\prime}}=\epsilon_{\perp}$, and $\epsilon_{j^{\prime} \neq k^{\prime}}=0$, where

$$
\begin{aligned}
\epsilon_{\perp}\left(\omega_{R}^{\prime}\right) & =\epsilon_{\mathrm{EIT}}\left(\omega_{R}^{\prime}\right) \\
& =\epsilon_{\infty}+\frac{A \gamma_{2 P}\left(\Delta-i \gamma_{1 S}\right)}{\left(\delta_{p}-i \gamma_{2 P}\right)\left(\Delta-i \gamma_{1 S}\right)-\Omega_{c}^{2} / 4},
\end{aligned}
$$

while $\epsilon_{\|}\left(\omega_{R}^{\prime}\right)$ is obtained by setting $\Omega_{c} \rightarrow 0$ in the above equation. Here $A \simeq 0.02$ is a numerical constant proportional to the $2 P$ exciton oscillator strength [7], $\epsilon_{\infty} \simeq 6.5+$ $i 2 \times 10^{-3}$ is the background dielectric constant, while $\Delta$ 
is the relative detuning $\left(\delta_{p}-\delta_{c}\right)$ with $\delta_{p}=\omega_{2 P}-\omega_{R}^{\prime}$ and $\delta_{c}=\omega_{2 P}-\omega_{1 S}-\omega_{c}^{\prime}$. Under EIT the dispersion equation for a probe (linearly) polarized in the $x^{\prime} y^{\prime}$ plane is that of an extraordinary ray [14] whose complex refractive index is given by

$$
\begin{aligned}
n^{2}\left(\omega_{R}^{\prime}, \chi\right) & =\frac{\epsilon_{\|}\left(\omega_{R}^{\prime}\right)}{1+\epsilon_{r}\left(\omega_{R}^{\prime}\right) \cos ^{2} \chi} ; \\
\epsilon_{r}\left(\omega_{R}^{\prime}\right) & \equiv \frac{\epsilon_{\|}\left(\omega_{R}^{\prime}\right)}{\epsilon_{\perp}\left(\omega_{R}^{\prime}\right)}-1 .
\end{aligned}
$$

Unlike in the absence of EIT for which $\epsilon_{r}=0$, not all variables involving the reduced dielectric function $\epsilon_{r}$ vanish, some variables acquiring smaller values than others for probe frequencies within the transparency bandwidth [7]. With the pump exactly resonant $\left(\delta_{c}=0\right), \operatorname{Re}\left[\epsilon_{r}\right]$ and the frequency derivatives of $\operatorname{Im}\left[\epsilon_{\|}\right]$and $\operatorname{Im}\left[\epsilon_{r}\right]$ take on vanishingly small values for a nearly resonant probe up to probe detunings of several tenths of $\gamma_{2 P}$ and a more compact form of the drag is obtained after setting these variables to zero in (4). The resulting form of $\alpha_{e}$ can be further simplified observing that in the same detuning range the remaining variables are nearly constant, acquire fairly larger values but still less than unit $\left(10^{-3} \div 10^{-2}\right)$, and increase according to $\left|\operatorname{Im}\left[\epsilon_{r}\right]\right|<\gamma_{2 P}\left|\partial_{\omega} \operatorname{Re}\left[\epsilon_{r}\right]\right| \leq \operatorname{Im}\left[\epsilon_{\|}\right] \lesssim$ $\gamma_{2 P}\left|\partial_{\omega} \operatorname{Re}\left[\epsilon_{\|}\right]\right|$. It is then possible to carry out a successive series expansion of $\alpha_{e}$ with respect to $\operatorname{Im}\left[\epsilon_{r}\right]$, $\gamma_{2 P} \partial_{\omega} \operatorname{Re}\left[\epsilon_{r}\right]$ and $\operatorname{Im}\left[\epsilon_{\|}\right]$to obtain, to the lowest order,

$$
\begin{aligned}
\alpha_{e} \simeq 1 & -\sqrt{\operatorname{Re}\left[\epsilon_{\|}\right]}-\frac{\omega_{I}}{2 \sqrt{\operatorname{Re}\left[\epsilon_{\|}\right.}} \\
& \times\left(\frac{\partial \operatorname{Re}\left[\epsilon_{\|}\right]}{\partial \omega_{I}}-\operatorname{Re}\left[\epsilon_{\|}\right] \cos ^{2} \chi \frac{\partial \operatorname{Re}\left[\epsilon_{r}\right]}{\partial \omega_{I}}\right) .
\end{aligned}
$$

Higher order contributions containing terms proportional to the product of $\operatorname{Im}\left[\epsilon_{r}\right], \operatorname{Im}\left[\epsilon_{\|}\right], \gamma_{2 P} \partial_{\omega} \operatorname{Re}\left[\epsilon_{\|}\right]$and of $\operatorname{Im}\left[\epsilon_{r}\right], \gamma_{2 P} \partial_{\omega} \operatorname{Re}\left[\epsilon_{r}\right], \operatorname{Im}\left[\epsilon_{\|}\right]$have been neglected and the frequency dependencies of all dielectric functions are not explicitly indicated.

The magnitude of $\alpha_{e}$ may be controlled directly by varying the intensity of the pump beam $\left(\Omega_{c}\right)$ and the cleavage angle $(\chi)$ so that the drag may acquire positive and negative values or it may even drop to zero. The first two terms on the right hand side of (7) are in fact of the same order of magnitude and much smaller than the last two in the bracket which are the dominant contributions to the drag. For appropriate choices of $\Omega_{c}$ 's and $\chi$ 's these two terms may also become comparable in magnitude so as to make the overall drag to vanish at probe frequencies which are determined by the specific selection of pump and cleavage parameters. For each probe detuning and set cleavage, there exists a pump intensity at which $\left|\alpha_{e}\right|$ is maximum and one for which $\alpha_{e}$ vanishes. Note, in particular, that the second of those terms does not appear in the absence of the pump beam, i.e., when $\epsilon_{\|}=\epsilon_{\perp}$ and $\epsilon_{r}=0$; the frequencies at which $\left|\alpha_{e}\right|$ is largest can no longer be tuned in this case but are fixed by the material parameters.
We report in Figs. 2 and 3 numerical results obtained from (7) for a resonant and a detuned pump. Because of the high dispersion at the $2 P$-exciton EIT resonance, where the absorption is quenched by quantum interference, large drags are associated with an appreciable degree of transparency in the region $\left|\delta_{p}-\delta_{c}\right|<0.5 \gamma_{2 P}$, where the observation of either large or vanishing drags should be favored. At exact resonance, $\alpha_{e}^{\max } \simeq 18$ occurs with over $20 \%$ transparency whereas $\alpha_{e}$ vanishes for slightly detuned probes with a little smaller transparency. These figures exceed those in the absence of the pump where, e.g., $\left|\alpha_{e}^{\max }\right| \simeq 10$ occurs with only a few percent
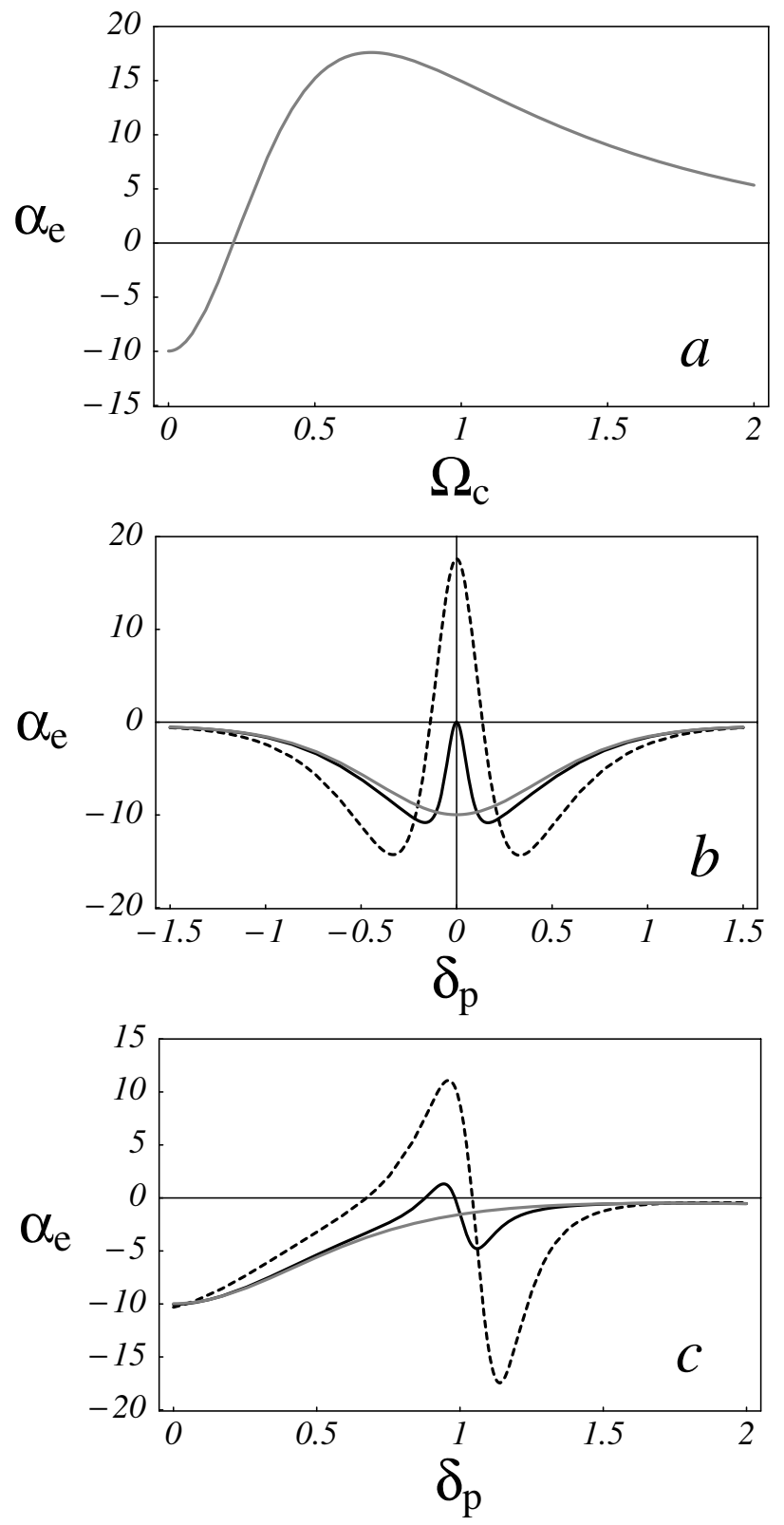

FIG. 2. Coefficient $\alpha_{e}$ vs $\Omega_{c}$ in units of $\gamma_{2 P}$ for a resonant probe (a). Coefficient $\alpha_{e}$ vs $\delta_{p}$ in units of $\gamma_{2 P}$ for a Rabi frequency of $\Omega_{c} / \gamma_{2 P}=0$ (grey line), 0.22 (solid line), and 0.7 (dashed line) for a resonant (b) and detuned (c) pump with $\delta_{c} /$ $\gamma_{2 P}=1$. Here the cleavage angle is $\chi=5^{\circ}$ and $\gamma_{2 P}=1 \mathrm{meV}$. 

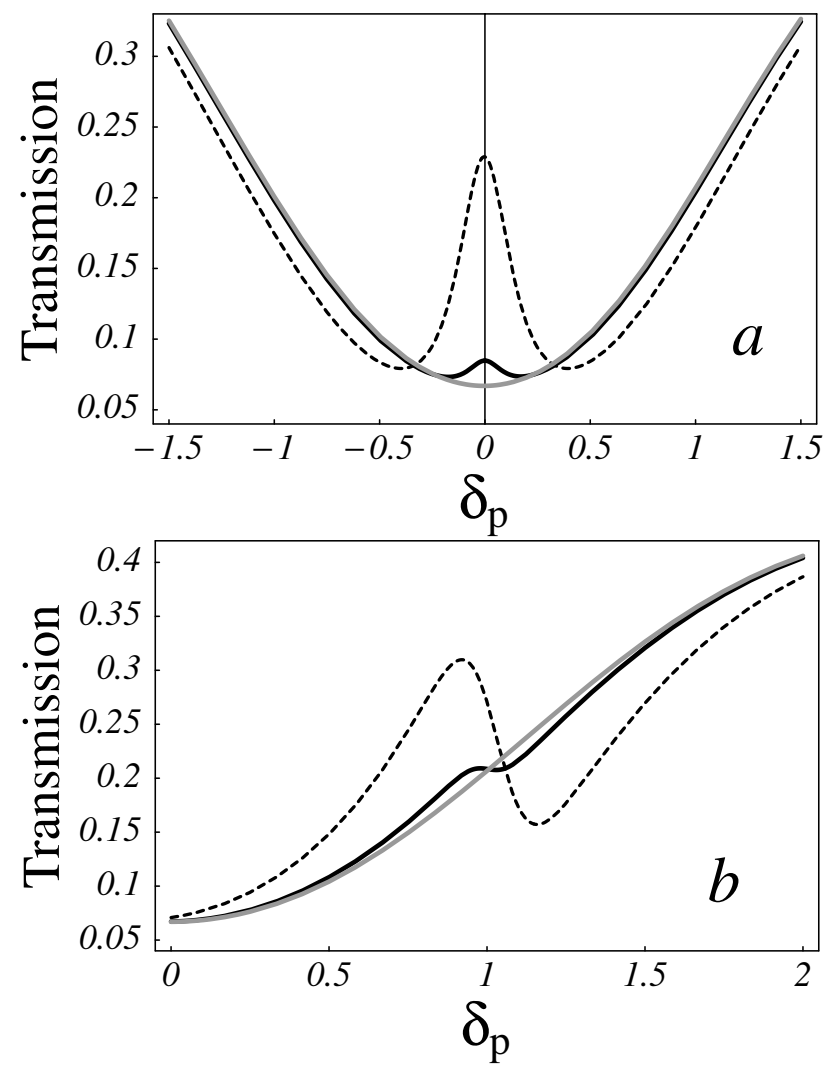

FIG. 3. Transmission coefficient for a resonant (a) and detuned (b) pump. The slab has a thickness $L^{\prime}=25 \mu \mathrm{m}$, while the other parameters are the same as in Fig. 2.

transparency. The situation improves further with the pump detuning: where $\alpha_{e}^{\max } \simeq 12$ with a $30 \%$ transparency, while zeros of $\alpha_{e}$ occur with a 20\% transparency.

A large $\alpha_{e}$ improves the accuracy of drag measurements since interferometric techniques measure $\left(\Delta \phi-\Delta \phi_{o}\right)$ that is proportional to the product $\beta \times \alpha_{e}[9,12]$. The present values of $\alpha_{e}$ exceed by at least 1 order of magnitude those for glass media [9]. At the $2 P$ exciton resonance $\partial_{\lambda_{p}} \operatorname{Re}\left[\epsilon_{\|}, \epsilon_{r}\right]$ varies in the range $10 \div 40 \mu \mathrm{m}^{-1}$ that is just about 1 to 2 orders of magnitude larger than typical dispersions for glass media [9]. Note that the dephasing $\gamma_{1 S}$ plays an important role. Reducing it to half its value would produce a further $35 \%$ increase in the magnitude of $\alpha_{e}$ with a concomitant $50 \%$ increase in the transparency window whose width would then narrow down to nearly a third of $\gamma_{2 P}$.

Our situation resembles much the one of neutron de Broglie waves in matter [15] where the dispersive contribution to the drag can compare with or exceed that determined by the refractive index of the medium. Matter waves and light waves in moving media share another and peculiar feature, i.e., the fact that the drag may vanish. In our case, in particular, the drag can vanish over a wide range of optical frequencies and such a remarkable behavior originates from the fact that around the $2 P$-exciton EIT resonance the two terms in the bracket of (7) may cancel with one another. To the best of our knowledge, null drags have so far been observed only for thermal neutrons traversing a moving medium contained inside stationary boundaries and exhibiting no nuclear resonance [8]. While it follows as a general result [16] that no neutron Fizeau effect occurs for an off resonance neutron probe and boundaries at rest, it is here worthwhile to note that in our case a null effect is instead exhibited for a light probe either on or off resonance and moving boundaries. The null result depends in one case on the specific form of the dispersion relation for neutrons in material media while on the other it depends both on the EIT dispersion and on the associated anisotropy of light waves in cuprous oxide.

In summary, we have theoretically investigated how electromagnetically induced transparency affects the Fresnel light drag, considering the yellow exciton line of cuprous oxide for specific numerical estimates. We have shown that the effective drag coefficient near resonance can be controlled with the pump intensity and detuning: it can acquire a wide range of values, positive or negative, and even vanishing.

We thank F. Cataliotti and S. Harris for enlightening discussions.

[1] A. Fresnel, Ann. Chim. Phys. 9, 57 (1818); 9, 286 (1818).

[2] L. D. Landau and E. M. Lifschitz, Electrodynamics of Continuous Media (Nauka, Moscow, 1982).

[3] H. Fizeau, C. R. Acad. Sci. B 33, 349 (1851); Ann. Chim. Phys. 57, 385 (1859).

[4] W. Rosser, An Introduction to the Theory of Relativity (Butterworths Press, London, 1964).

[5] S. Harris, Phys. Today 50, No. 7, 36 (1997); E. Arimondo, Progress in Optics $X X X V$, edited by E. Wolf (Elsevier Science, Amsterdam, 1996), p. 257, and references therein.

[6] G. Alzetta, A. Gozzini, L. Moi, and G. Orriols, Nuovo Cimento Soc. Ital. Fis. 36B, 5 (1976); E. Arimondo and G. Orriols, Lett. Nuovo Cimento 17, 333 (1976).

[7] M. Artoni, G. C. La Rocca, and F. Bassani, Europhys. Lett. 49, 445 (2000).

[8] M. Arif et al., Phys. Rev. A 31, 1203 (1985).

[9] G. A. Sanders et al., J. Opt. Soc. Am. B 5, 674 (1988), and references therein.

[10] P. Zeeman, Proc. R. Acad. Sci. Amsterdam 17, 445 (1914); 18, 398 (1915).

[11] For tilted samples additional aberration effects should be considered which would lead to a more complex form of the shift (4).

[12] W. Macek et al., J. Appl. Phys. 35, 2556 (1964).

[13] J. Jackson, Classical Electrodynamics (J. Wiley, New York, 1975), 2nd ed.

[14] M. Born and E. Wolf, Principles of Optics (Pergamon Press, Oxford, 1993).

[15] A. Klein et al., Phys. Rev. Lett. 46, 1551 (1981).

[16] M. Horne et al., Phys. Rev. A 28, 1 (1983). 